\title{
Impacto de las TIC en el nivel de innovación en América Latina y el Caribe: Estimaciones econométricas a nivel de un panel
}

Karol Rodriguez Cabrera, Jorge Andrés Vélez Ospina

\begin{abstract}
Resumen
La contribución de las Tecnologías de la Información y las Comunicaciones (TIC) como jalonador principal de la innovación y este a su vez como fuente de crecimiento y desarrollo económico, ha tomado gran importancia en la agenda pública mundial; por su parte los países de América Latina, se han preocupado por incentivar el uso y apropiación de TICS no sólo por la inserción en la dinámica global sino por el impacto en el mejoramiento de los procesos productivos en todos los sectores. En ese sentido, esta investigación propone una aproximación empírica para evaluar el impacto de las TIC en el nivel de innovación en América Latina y el Caribe, considerando la existencia de factores económicos de ambiente de negocios e institucionales que contribuyen en el desarrollo de actividades de innovación, para ello se elabora un modelo econométrico de panel de datos para una muestra de 20 países de América Latina en el periodo 2000-2011, se espera que un incremento en el uso de TICs mejore los niveles de innovación de los países.
\end{abstract}

Palabras claves: Tecnologías de la Información y las Comunicaciones, Innovación, Panel de datos, derrames tecnológicos. JEL Classification: L80,L96, 014. 


\section{Introduccion}

Las diferencias de ingreso y crecimiento entre los países de América Latina y el Caribe, corresponden a diferencias en la productividad total de los factores. Esa diferencia puede estar marcada por el progreso tecnológico y el nivel de innovación (Perry, 2005; DeFerranti y otros, 2005). Por lo tanto, los cambios técnicos y el nivel de innovación de la empresa y en agregado son determinantes a la hora de visualizar la senda competitiva y de crecimiento de una economía.

Cabe resaltar que este proceso de innovación no se da de manera espontánea, en el mismo convergen condiciones financieras, institucionales y las diferentes decisiones de los empresarios para sostenerse en el mercado de manera competitiva. Además de estas variables, existen otro tipo de determinantes que explican los procesos de innovación. Por ello, este documento trata de capturar el impacto que tienen los procesos de apropiación y utilización de las Tecnologías de la Información y las Comunicaciones (TIC) para los procesos de innovación a nivel de un panel de países. En ese sentido, se desarrolla un modelo econométrico utilizando la técnica de panel de datos para una muestra aleatoria de países de América Latina y el Caribe, que trata de explicar si la transferencia de TIC y los derrames tecnológicos derivados de las TIC (spillovers), han impactado la dinámica de innovación para el periodo 2000 - 2011. Se espera que un mayor uso de TIC y los derrames tecnológicos de la región generen efectos positivos y significativos en los niveles de innovación para estos países. Sin embargo, en los países de América Latina y el Caribe los altos niveles de Inversión Extranjera Directa (IED), no siempre responden a tasas altas de transferencia de tecnología en los procesos de innovación.

A pesar de que no exista una relación deterministica entre el esfuerzo tecnológico (en TIC), la innovación, productividad y crecimiento económico de un país, existen indicios de que el carácter del cambio tecnológico intensivo en destrezas se ha acentuado en las recientes décadas, en parte como resultado de la denominada revolución de las TIC (DeFerranti y otros, 2005), en tal medida, se reconoce que la apropiación y el uso de las TIC han afectado todos los aspectos de la dinámica económica, transformando no sólo el modo en que se llevan a cabo las actividades productivas, sino también las decisiones de innovación a nivel de cada uno de los sectores económicos de un país. En este sentido, este trabajo de investigación, propone una aproximación empírica al problema de evaluar el impacto del uso de las TIC en el nivel de innovación de cada país. Para ello se parte de los planteamientos teóricos de la economía de la innovación y su relación con los desarrollos tecnológicos a nivel de TIC.

La organización de este trabajo es como sigue. En el primer capítulo se explican las interacciones entre el proceso de innovación y TIC, haciendo un contraste de los principales trabajos teóricos y empíricos que han abordado dicha relación. En la segunda parte se especifica el modelo econométrico, donde se introducen dos aspectos novedosos. En primer lugar, se estima la ecuación de innovación que relaciona variables de tipo institu- 
cional y del ambiente tecnológico (TIC), adicional a ello se adhieren variables de control de tipo económico de negocios e institucional para realizar innovaciones. Por último, en el capítulo tres se presentan los hechos estilizados, estimaciones y pruebas econométricas, para brindar finalmente las respectivas conclusiones y recomendaciones de política.

Metodológicamente este tipo de acercamiento permite evidenciar la forma en la cual el desarrollo tecnológico derivado de las TIC ha dado espacio para establecer efectos positivos en el nivel de innovación en la medida en que ha reorientado la dinámica económica de los países. Lo cual permite diseñar las bases para la formulación de políticas de fomento empresarial e incentivos al uso de TIC en la esfera productiva.

\section{Revisión de literatura}

\section{REVISIÓN DE LITERATURA A NIVEL TEÓRICO Y EMPÍRICO}

La literatura económica ha abordado desde diferentes perspectivas la relación entre TIC e innovación, entre ellas se encuentra la concepción de cambio tecnológico de Schumpeter (1947), en donde las TIC son consideradas como una herramienta necesaria para intensificar la productividad multifactorial entendida como aquella productividad no añadida por los factores capital y trabajo, de alli que Brynjolfsson (1997) afirma que la contribución conjunta de las TIC y las innovaciones tecnológicas es clave para el crecimiento y competitividad de las economías.

En esa misma línea, la teoría económica parte de los planteamientos de la tradición neoclásica, donde el progreso técnico se asocia a la productividad total de los factores (PTF) o productividad multifactorial y su relación con el crecimiento económico (Solow, 1956; Swan, 1956; Harrod 1949) y simultáneamente a los trabajos de la denominada teoría endógena del cambio técnico (Romer, 1986 y 1990; Mankiw, Romer y Weil, 1992; Grossman y Helpman (1991). A partir de estos planteamientos, trabajos como los de Tebaldi y Elmslie (2007), Acemoglu y otros (2001) y (2004), Easterly y Levine (2003), formalizan modelos de crecimiento económico donde se evalúa la influencia de las instituciones en el desempeño económico, constatando los efectos de éstas en la inversión de tecnologías TIC y el crecimiento económico.

En efecto, una de las variables que dinamiza el crecimiento económico de los países es el nivel de emprendimiento empresarial como lo han demostrado los trabajos de Morris (2001); Carrée y Thurik (2003); Stel (2005). Por lo tanto es clave analizar las relaciones derivadas entre las TIC y el nivel de innovación de un país, dado que es alli donde se derivan las relaciones en el largo plazo entre crecimiento económico y TIC. Además frente a las crecientes preocupaciones sobre el crecimiento económico y la competitividad en los mercados mundiales, los gobiernos han respondido a esta nueva evidencia estimulando las políticas de innovación (Audretsch y Thurik, 2001, OCDE, 1998). 
De esta forma, literatura reciente sobre los determinantes de la innovación a nivel de los países, se ha centrado en analizar las variables económicas que influyen en el nivel de innovación, bajo esta línea se resaltan los trabajos de Carree y Thurik (2003); Geroski y Jacquemin (1985); OECD (1998)'1.

Por su parte, Sapprasert (2007) explora empíricamente el vínculo sólido entre las TIC y el crecimiento de los servicios en Noruega. Su principal objetivo es investigar la contribución conjunta de las TIC en el fomento de la innovación de servicios. El trabajo demuestra que las TIC han logrado incrementar la competitividad y el crecimiento del sector servicios. Este estudio revela que la mayoría de las empresas de servicios cuya I+D supera el promedio industrial, han experimentado un mayor crecimiento. Estos corroboran el papel de las TIC como uno de los motores principales que conducen al desempeño económico superior de las industrias de servicios [Gershuny y Miles, 1983; Freeman y Louca, 2002]. Así se demuestra que los coeficientes de intensidad entre las TIC, la productividad y la rentabilidad son positivos y estadísticamente significativos para las empresas de servicios, mientras que menos significativas para las empresas de la industria manufacturera. [Bresnahan y Trajtenberg, 1995; Bresnahan et al, 2002.], hay que resaltar que en este estudio se incluyen las innovaciones no tecnológicas con el fin de corroborar la contribución efectiva de las TIC en el sector servicios.

En una perspectiva histórica, Freeman (2002) explora cómo los países logran alcanzar tecnológicamente a otros. Argumenta que el éxito está en los sistemas de innovación Nacional. Este es el caso de Inglaterra, EE.UU., Japón y, más recientemente, los tigres asiáticos. Uno de los estudios que explora las relaciones entre innovación y TIC es el de Pavitt and Soete (1981) y Broersma (2007), ellos encuentran una relación positiva entre el nivel de las TIC y la productividad de las empresas. Las pruebas apuntan a diferentes factores para moderar el impacto de las TIC a nivel de empresa, incluyendo la co-ocurrencia de adecuación de las capacidades de la fuerza laboral, una práctica organizativa adecuada y otras formas de innovación tecnológica. Por otra parte, el tamaño y la antigüedad de la empresa parece influir en el efecto de la adopción de las TIC en la productividad.

De otro lado, aunque las mejoras en la tecnología de TI tienden a adoptarse de manera muy rápida, los cambios organizativos complementarios a nivel de empresa se basan en un proceso de "co-invención" de las empresas individuales (Bresnahan y Greenstein, 1997), sugiriendo co-evolución como aquellos procesos que combinan 1) la adopción de tecnologías de la información, 2) cambios organizacionales complementarios, y 3) la innovación en la forma de nuevos productos y servicios, (Bresnahan, Brynjolfsson y Hitt (2002). Esto indicaría que existe cierto grado de complementariedad entre las TIC y la Innovación.

De otro lado, Bloom y otros (2011) usando datos microeconómicos a nivel de empresa, una de sus exploraciones se deriva en buscar los efectos secundarios de las TIC

1 Estos trabajos analizan variables como el nivel de ingreso, el desempleo, los niveles educativos, el costo de capital, y algunas variables demográficas. Otros trabajos como los de Wennekers et al (1999) analizan factores culturales e institucionales determinantes del nivel de innovación. 
al modo como se analizan los derrames o spillover de I+D (Griliches, 1992; Jaffe 1986, Bloom, Schankerman y Van Reenen 2009). Entienden el spillover de TIC como aquel en el cual unas empresas están más cerca a otras.

En ese sentido, la siguiente expresión denota el Spillover como una nueva variable que mide la intensidad de uso de las TIC a lo largo de una serie de dimensiones posibles.

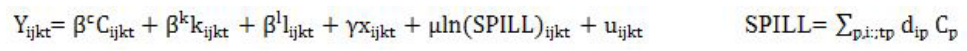

Las distancias (d) se basan en la industria, por ejemplo, todas las demás empresas no pertenecientes a la industria se les da un peso de uno $(\mathrm{dip}=1)$, mientras que las empresas pertenecientes a la industria se ponderan cero $(\operatorname{dip}=0)$.

Si los spillovers vienen de eslabonamientos hacia adelante o hacia atrás, podrían ser usadas matrices de insumo-producto o matrices comerciales.

A su vez, Sanchez y Zayas (2008) estudian la innovación en los servicios de la Unión Europea (UE), analizando dos dimensiones : la densidad de la innovación y la preeminencia económica de los innovadores, entendiéndose la primera como la realización de innovaciones de una empresa en un sector determinado, y la segunda como el valor económico de dichas innovaciones, se consideran todos los países de la UE a excepción de Irlanda, Noruega e Islandia, en la constatación se realiza un análisis factorial tomando datos de la Encuesta de Innovación (CIS3) periodo (1998 - 2001), es de resaltar que en este estudio se introduce una variable reconociendo el dinamismo innovador diferenciador entre sectores y países.

En efecto, se reconoce que las variables significativas en el análisis son aquellas que se refieren al sector y pais de pertenencia, de manera que las empresas encuentran mayores incentivos para innovar si pertenecen a un grupo.

Por otra parte, el estudio denota que los casos en los que se tiene menor influencia del dinamismo del pais son indicativos de que la explotación comercial de la tecnología es menos complicada que su generación, siendo por tanto extraordinariamente relevante la capacidad de absorción.

Una consideración relevante en términos de política señala que los mecanismos de protección estándar no son los más efectivos en el sector servicios. En ese sentido, el copyright y las formas de protección tradicional no contribuyen a mejorar la preeminencia económica de los innovadores y reducen la densidad de la innovación. En esa misma línea, se afirma que la realización de actividades de innovación tecnológica distintas de la I+D parecen favorecer tanto la densidad de innovación como la preeminencia económica de los innovadores, dado que las empresas utilizan mecanismos y acciones de innovación, en muchos casos, alternativos a los tradicionalmente empleados en las manufacturas; en especial dicho efecto es atribuible a la preocupación por la percepción del producto por los consumidores y las acciones de comercialización del mismo. 
Figura 1. Determinantes de la Innovación

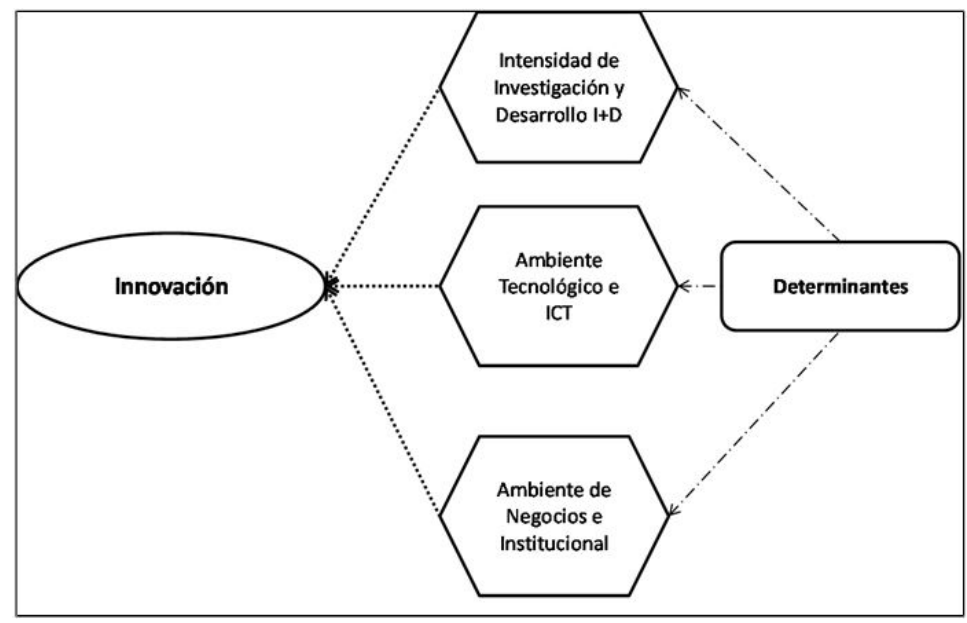

Ahora bien, Maloney y Perry (2005) dando una mirada a la política de innovación en América Latina, reconocen que las diferencias de ingreso y crecimiento entre los países corresponden básicamente a diferencias en la productividad total de los factores (PTF) , que dichas diferencias se evidencian en el progreso tecnológico y a su vez en la capacidad de innovación, en donde sin duda, cambios técnicos, institucionales, orgánicos y administrativos y el desarrollo de actividades y productos nuevos en los niveles micro y macro de una economía juegan un papel fundamental.

En ese sentido, los países de América Latina han estado marcados por la explotación de recursos naturales como fuente principal de ingresos, lo cual desató bajos niveles de crecimiento e inconvenientes en el proceso de industrialización, quizá por falta de conocimientos y por una dependencia pasiva de transferencia de tecnología. Dicho contexto no ha favorecido los procesos de innovación en la región, de allí que se noten bajos niveles de inversión en I+D, falta de participación en I+D en el sector privado, y que el peso relativo de la investigación básica sea mayor que el de la investigación aplicada.

Por consiguiente, los autores consideran que la innovación merece atención especial en la agenda política en América Latina, que en estricto sentido no se puede hacer una formulación acertada de política de innovación si se desconocen los verdaderos problemas que inciden en el fomento de la misma. En términos generales, Maloney y Perry (2005) anotan que para estimular la innovación se deben concentrar los esfuerzos en desarrollar la capacidad y los incentivos de las empresas, pues si no hay demanda de innovación por el sector privado, serán ineficaces los esfuerzos por mejorar la capacidad científica y tecnológica por el lado de la oferta, bajo la premisa 
de que la productividad que a su vez se evidencia en el progreso tecnológico y en la capacidad de innovación es un fenómeno que depende principalmente de las empresas.

\section{Modelo de impacto de las TIC en el nivel de innovación: esquema metodológico}

\section{ESPECIFICACIÓN ECONOMÉTRICA DEL MODELO}

Para realizar las estimaciones econométricas pertinentes, se plantea el siguiente modelo, en el cuál, se incorporan aquellos factores explicativos más relevantes en la función de innovación. El modelo de panel de datos, utiliza datos para 20 países para los periodos 2000 a 2011 tomados del Banco Mundial y algunas otras fuentes estadísticas. Así, la especificación se adapta a la forma funcional presentada en la ecuación (1).

$$
I_{i, t}=a+X^{i t} f 3+u^{i, t}
$$

Donde la variable determinada $\hat{l}_{t, i}$ se considera dos variables asociadas al nivel de innovación. A su vez la muestra de países corresponde al subíndice $i\{i=1,2,3 \ldots$ $20\}$ y $\{t=2000,2001, \ldots, 2011\}$ es la dimensión temporal del panel, a es un escalar, $\mathrm{f} 3$ es un vector de $\mathrm{K} \times 1$ y $\mathrm{X}$ es la i-th observación sobre $\mathrm{K}$ variables explicativas sobre el entorno TIC y el entorno económico institucional.

Cabe observar que el componente de error toma la forma de la ecuación (2).

$$
\mathrm{u}_{\mathrm{it}}=\mu_{\mathrm{i}}+\mathrm{v}_{\mathrm{i}, \mathrm{t}}
$$

En (2) $\mu \mathrm{i}$ : representa los efectos no observables que difieren entre las unidades de estudio pero no en el tiempo, que generalmente se los asocia a la capacidad de cada país para innovar, por ejemplo; $v_{i, t}$ se refiere al término de error puramente aleatorio, es decir, se distribuye con media cero y varianza constante, formalmente: $v_{i, t} \sim N\left(0, c^{2}\right)$.

Como se indicó brevemente, una posibilidad es explicar los datos con el modelo de efectos fijos, donde se considera que existe un término constante diferente para cada individuo, y supone que los efectos individuales son independientes entre sí. Con este modelo se considera que las variables explicativas afectan por igual a las unidades de corte transversal y que éstas se diferencian por características propias de cada una de ellas, medidas por medio del intercepto. Por ello, desde el punto de vista teórico se va asumir que el componente $\mu \mathrm{i} \neq 0$ es decir, se considera efectos 
fijos y específicos para cada país, en este caso, la heterogeneidad no observable se incorpora a la constante del modelo. Reescribiendo (1) y teniendo en cuenta el efecto fijo, tenemos la especificación 5.

$$
I_{i, t}=\alpha+X_{i, t} \beta+u_{i}+v_{i t}
$$

Promediando para todo el periodo se obtiene (6).

$$
\bar{I}_{i, t}=\alpha+\bar{X}_{i, t} \beta+u_{i}+\bar{v}_{i}
$$

Y tomando para toso los individuos se obtiene la ecuación (7)

$$
\bar{I}=\alpha+\bar{X} \beta+\bar{v}
$$

En (7) el vector de innovación: ${ }^{\overline{1}}=\sum_{i=1}^{\mathrm{N}} \mathrm{I}_{\mathrm{i}, t} / \mathrm{NT}$, , similarmente $\overline{\mathrm{X}}=\sum_{\mathrm{i}=1}^{\mathrm{N}} \mathrm{X}_{\mathrm{i}, t} / \mathrm{NT}$. Obsérvese que en (7) el efecto fijo $u_{i}$ suma cero.Definiendo de manera similar, se obtiene las ecuaciónes (8) y (9).

$$
\begin{aligned}
& I_{i, t}=\left(I_{i, t}-\bar{I}\right)=\beta\left(X_{i, t}-\bar{X}\right)+\left(v_{i, t}-\bar{v}\right) \\
& I_{i, t}=\tilde{X}_{i, s} \beta+\tilde{v}_{i t}
\end{aligned}
$$

Corriendo el modelo (9) por Mínimos Cuadrados Ordinarios (MCO), se encuentran los parámetros del modelo de innovación asumiendo efectos fijos. El modelo expresado en (9) también es conocido como el estimador Within.

Para la i-ésima unidad de corte transversal, la relación sugerida se expresa en (10), esta ecuación pretende estimar los efectos de variables de uso y apropiación de TIC y otras variables a nivel económico e institucional y su relación con el nivel de innovación por país. Para este caso, los vectores $\mathrm{TIC}_{t, i}$ y $F_{i}$ incluyen los diferentes determinantes y que varian para cada país. Las variables control están especificadas en la tabla 1.

$$
\tilde{J}_{i, t}=\alpha+\sum_{k=1}^{K} \beta_{k, i,} T I C_{i, t}+\sum_{k=1}^{K} \beta_{k, j, t} F_{i,}+\tilde{v}_{i t}
$$

Donde:

$\mathrm{TIC}_{\mathrm{t}, \mathrm{i}}=$ Vector que relaciona las variables de tecnologias de información y las comunicaciones. Se espera que la absorción el nivel de innovación para el panel de países aumente con el nivel de uso y apropiación de TIC. (Ver Tabla 1). $F_{t, i}=$ Vector que refleja variables de control que reflejan el entorno institucional y económico por pais (Ver Tabla 1). 
Tabla 1. Ecuaciones del modelo de determinantes de innovación

\begin{tabular}{|c|c|c|c|c|}
\hline & & VARIABLES & DESCRIPCIÓN & FUENTE \\
\hline & & $\begin{array}{l}\text { Patentes Otorgadas } \\
\text { Residentes }\end{array}$ & $\begin{array}{l}\text { Número de patentes otorgadas efectivamente a residentes en } \\
\text { una oficina nacional de patentes por los derechos exclusivos } \\
\text { sobre un invento: un producto o proceso que presenta una } \\
\text { nueva manera de hacer algo o una nueva solución técnica a un } \\
\text { problema. Una patente brinda protección respecto de la } \\
\text { invención al dueño de la patente durante un periodo limitado } \\
\text { que suele abarcar } 20 \text { años. }\end{array}$ & $\begin{array}{l}\text { Red de Indicadores } \\
\text { De Ciencia y } \\
\text { Tecnologia } \\
\text { Iberoamericana e } \\
\text { Interamericana }\end{array}$ \\
\hline \multirow{5}{*}{ 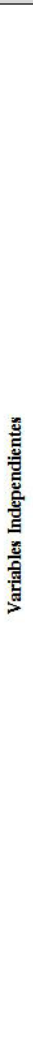 } & \multirow{3}{*}{ 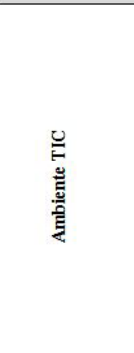 } & Banda Ancha & $\begin{array}{l}\text { Subscritores de banda ancha (por cada } 100 \text { habitantes). Se } \\
\text { describe como el número total de suscriptores de banda ancha } \\
\text { con una línea digital, módem por cable u otras tecnologías de } \\
\text { alta velocidad. }\end{array}$ & $\begin{array}{l}\text { World Development } \\
\text { Indicators Online. } \\
\text { 2008. World Bank }\end{array}$ \\
\hline & & $\begin{array}{l}\text { Computadores } \\
\text { personales por cada } \\
100 \text { personas } \\
\text { (PC) }\end{array}$ & $\begin{array}{l}\text { Número de ordenadores personales que se utilizan por cada } \\
100 \text { habitantes. }\end{array}$ & $\begin{array}{l}\text { Millennium } \\
\text { Development Goals } \\
\text { Database. United } \\
\text { Nations Statistics } \\
\text { Division }\end{array}$ \\
\hline & & $\begin{array}{l}\text { Celulares por cada } \\
100 \text { personas } \\
\text { (Cellphone) }\end{array}$ & Número de Celulares por cada 100 habitantes. & $\begin{array}{l}\text { Millennium } \\
\text { Development Goals } \\
\text { Database. United } \\
\text { Nations Statistics } \\
\text { Division }\end{array}$ \\
\hline & $\begin{array}{l}\text { Investigación y } \\
\text { Desarrollo }\end{array}$ & $\begin{array}{l}\text { Intensidad de } \\
\text { Investigación y } \\
\text { Desarrollo (I+D) } \\
\text { como porcentaje del } \\
\text { PIB }\end{array}$ & $\begin{array}{l}\text { Los gastos en investigación y desarrollo son gastos corrientes y } \\
\text { de capital (público y privado) en trabajo creativo realizado } \\
\text { sistemáticamente para incrementar los conocimientos, incluso } \\
\text { los conocimientos sobre la humanidad, la cultura y la sociedad, } \\
\text { y el uso de los conocimientos para nuevas aplicaciones. E1 área } \\
\text { de investigación y desarrollo abarca la investigación básica, la } \\
\text { investigación aplicada y el desarrollo experimental. } \\
\text { E1 gasto esta medido como \% del PIB. }\end{array}$ & $\begin{array}{l}\text { The Index of } \\
\text { Economic Freedom, } \\
\text { Heritage Foundation }\end{array}$ \\
\hline & 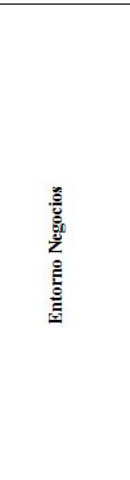 & $\begin{array}{l}\text { Facilidad de Hacer } \\
\text { Negocios }\end{array}$ & $\begin{array}{l}\text { Medida cuantitativa de la rigurosidad, cantidad y costo de los } \\
\text { requisitos para empezar, operar, y cerrar un negocio. El indice } \\
\text { es baso en } 10 \text { factores todos ponderados por igual usando } \\
\text { datos del World Bank doing Bussines. } \\
\text { E1 índice se construye como el promedio simple de los países } \\
\text { clasificación percentil de } 10 \text { temas: Creación de una empresa, } \\
\text { Manejo de licencias, empleo de trabajadores, registro de } \\
\text { propiedades, obtención de crédito, protección de inversores, } \\
\text { pago de impuestos, comercio transfronterizo, cumplimiento de } \\
\text { contratos y cierre de una negocio. } \\
\text { Las economías están clasificadas en la facilidad de hacer } \\
\text { negocios, de } 1 \text { (más fácil) a } 183 \text { (más dificil). Una buena (baja) } \\
\text { puntuación en el indice de facilidad para hacer negocios } \\
\text { significa el entorno normativo propicio para la operación del } \\
\text { negocio. E1 indice se construye como el promedio simple de } \\
\text { los paises clasificación percentil de } 10 \text { temas: compuestos por } \\
\text { una variedad de indicadores, dando el mismo peso a cada tema. } \\
\text { Las clasificaciones de } 2009 \text { son de Doing Business 2010: } \\
\text { Reformar en tiempos dificiles, que abarca el periodo } \\
\text { comprendido entre junio de } 2008 \text { hasta mayo de } 2009 \text {. }\end{array}$ & $\begin{array}{l}\text { World Economic } \\
\text { Outlook Database } \\
\text { The International } \\
\text { Monetary Fund. }\end{array}$ \\
\hline & \multirow{2}{*}{ 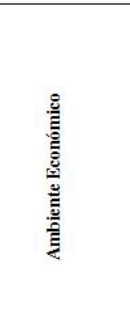 } & $\begin{array}{l}\text { Crédito interno al } \\
\text { sector privado }\end{array}$ & $\begin{array}{l}\text { El crédito interno al sector privado se refiere a los recursos } \\
\text { financieros prestados al sector privado a través de préstamos, } \\
\text { compras de valores, créditos comerciales y otras cuentas por } \\
\text { cobrar, que establecen una solicitud de devolución. En algunos } \\
\text { paises estas afirmaciones incluyen el crédito a las empresas } \\
\text { públicas. }\end{array}$ & $\begin{array}{lr}\text { World } & \text { Economic } \\
\text { Outlook } & \text { Database } \\
\text { The International } \\
\text { Monetary Fund. }\end{array}$ \\
\hline & & $\begin{array}{l}\text { Grado de Apertura } \\
\text { comercial }\end{array}$ & $\begin{array}{l}\text { La relación comercio-PIB se utiliza frecuentemente para medir } \\
\text { la importancia de las transacciones internacionales relativos a } \\
\text { las transacciones internas. Este indicador se calcula para cada } \\
\text { pais, como el promedio simple (es decir, la media) del total del } \\
\text { comercio (es decir, la suma de las exportaciones e } \\
\text { importaciones de bienes y servicios) en relación con el PIB. } \\
\text { (Definición de OECD Library) }\end{array}$ & $\begin{array}{lr}\text { World } & \text { Economic } \\
\text { Outlook } & \text { Database } \\
\text { The International } \\
\text { Monetary Fund. }\end{array}$ \\
\hline
\end{tabular}




\section{Resultados}

RELACIONES ENTRE TIC, INDICADORES DE AMBIENTE DE NEGOCIOS E INSTITUCIONAL SOBRE EL NIVEL DE INNOVACIÓN

Figura 2. Relación entre el nivel de TIC y el nivel de patentes otorgadas residentes promedio América Latina

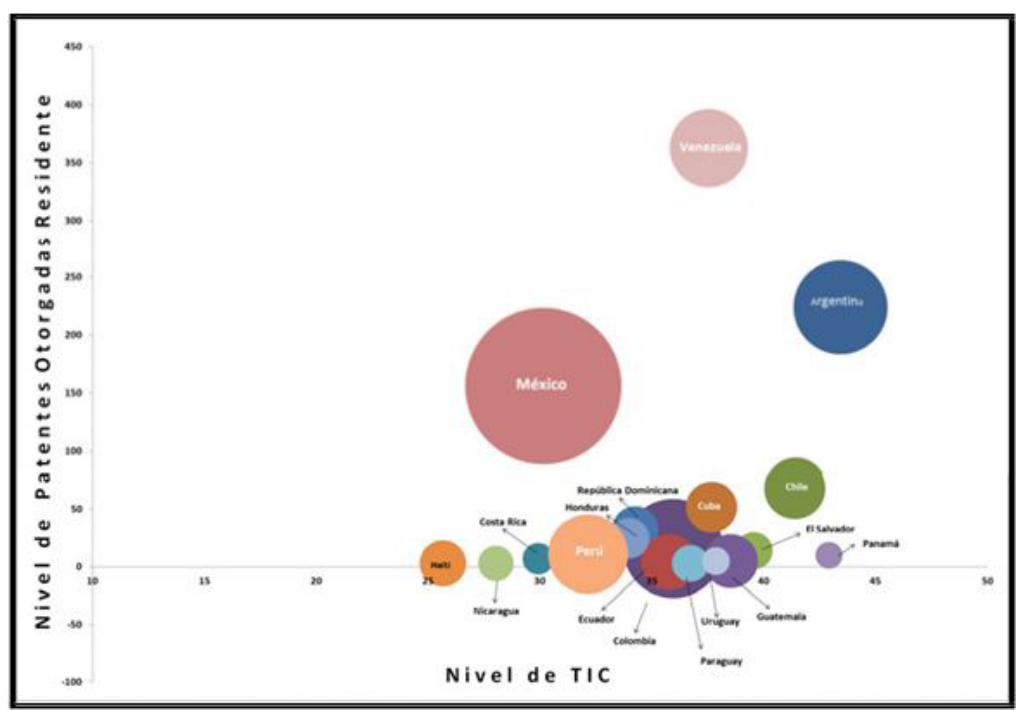

En la Figura 2 se presenta la relación entre el nivel de TIC y las patentes otorgadas a residentes como promedio en el periodo 2000-2011 para América Latina, se puede notar que países como México, Venezuela, Argentina, Chile y Cuba han tenido dinámicas favorables en innovación combinadas con el incremento en el nivel de TIC, México por su parte ha sido ejemplo de la consolidación de políticas institucionales hacia el fortalecimiento del sistema de Ciencia, Tecnología e Innovación, y en esa medida ha diseñado mecanismos para incentivar gradualmente la protección de las innovaciones. Sin embargo, en países como Paraguay, Uruguay, Guatemala y Ecuador, aunque se han hecho esfuerzos por incrementar el uso y apropiación de TIC, aún existen iniciativas desarticuladas que no han tenido mayor impacto sobre los niveles de innovación.

Tal y como se nota en la Figura 3 a y b el nivel de TIC y las patentes otorgadas en América Latina en el periodo 2000-2011 han tenido una tendencia similar, Io cual representa una aproximación a la relación que existe entre estas variables. 
Figura 3. (a) (b). Nivel de TIC promedio América Latina (a) y Nivel de patentes otorgadas promedio América Latina (b)

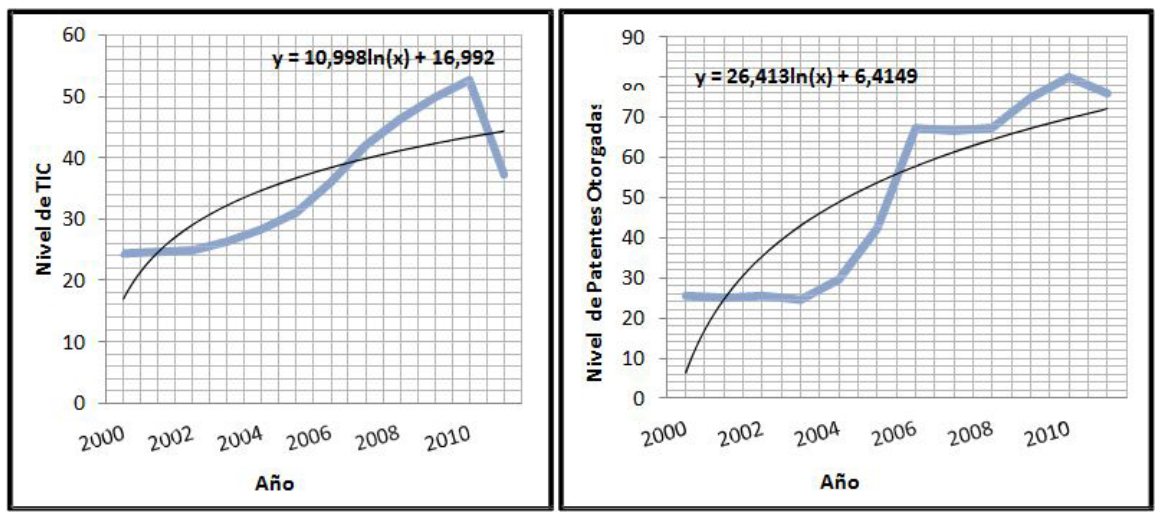

Vale la pena anotar que a pesar de existir pocos indicadores de producción tecnológica disponibles, las patentes resultan ser la variable proxy más utilizada para medir los niveles de innovación, dado que permiten calcular la capacidad innovadora en un país.

Por lo anterior, al observar la dinámica de las patentes otorgadas en América Latina se nota que esta ha venido incrementándose favoreciendo los niveles de innovación en la región, dicho incremento no se ha dado de manera desarticulada, con ella se han combinado aspectos de tipo tecnológico, económico e institucional: desde la divulgación de las autoridades en propiedad industrial sobre los derechos de innovación, los acuerdos regionales para la protección de la propiedad industrial en varios países y el aumento de las capacidades tecnológicas de las empresas hasta las medidas de regulación económica incentivando la competencia en los mercados.

En los países latinoamericanos los incrementos en el nivel de patentes se ha dado no sólo en el otorgamiento de patentes a residentes sino a No residentes, según Ia SIC (2012), en estadísticas de la Organización Mundial de la Propiedad Intelectual en los paises desarrollados más del $50 \%$ de solicitudes de patente son realizadas por residentes del país. Sin embargo, en países emergentes este porcentaje es de apenas el 20\% en promedio.

Variable Determinada: Un análisis del nivel de innovación y su relación con las variables explicativas del modelo, muestra que la difusión de las tecnologías de la información y las comunicaciones (TIC) influye de manera positiva sobre el nivel de innovación de los países.

Se puede observar en las figuras $(4,5 a$ y $5 b)$ que el nivel de suscriptores a banda ancha fija e internet, a telefonía móvil celular y el uso de computadores 
personales, tienen una relación positiva (tendencialmente) frente al nivel de patentes otorgadas. Es decir, el uso de TIC se traduce en mejora de tiempos y acceso a información, lo que le permite a las empresas tomar decisiones más acertadas de manera que favorece la capacidad de las mismas para desarrollar nuevos procesos productivos que los hagan más competitivos en los mercados.

Figura 4. Suscriptores a telefonía móvil celular y su relación con el nivel de innovación

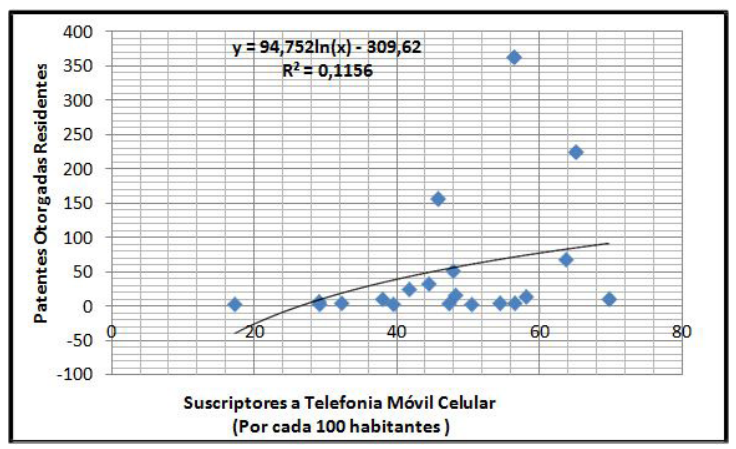

De otro lado, se observa una relación levemente positiva entre las patentes otorgadas y el número de suscriptores a telefonía móvil celular (Figura 3). De tal manera que, el acceso a telefonía móvil celular incide en la capacidad de las empresas para desarrollar nuevos procesos productivos o mejorar los existentes de modo que se incrementan los niveles de innovación, de hecho cifras del Banco mundial (2012), muestran que en el 2011 el número de abonados a telefonía celular en América Latina representaba el 10,65\% de los abonados mundiales.

Figura 5 (a) (b). Suscriptores a banda ancha fija (a) y Computadores Personales por cada 100 habitantes y su relación con el nivel de Innovación
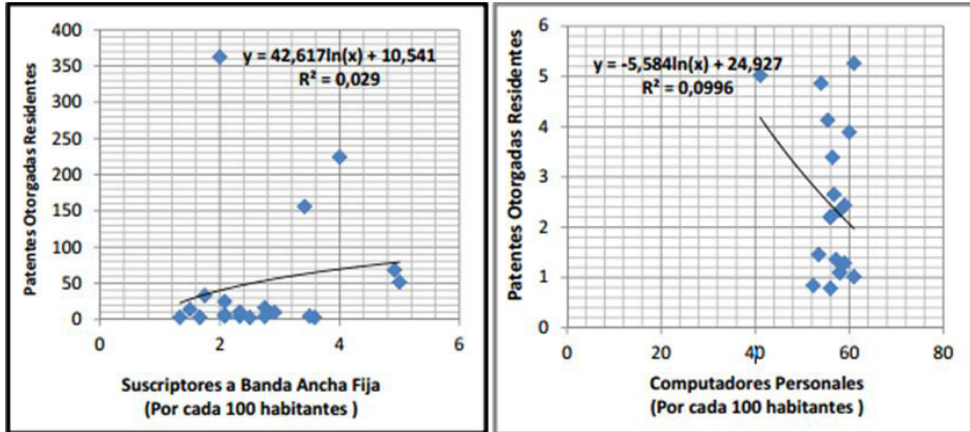
En lo referente a los suscriptores de banda ancha fija e internet y el uso de computadores personales (Figuras 5 a y 5 b), es importante anotar que dichas herramientas se han convertido en elementos esenciales en el desarrollo de cualquier proceso económico, y en esa medida su uso ha contribuido en el incremento de las actividades de innovación y desde luego en el nivel de patentes.

En cuanto a la posible relación entre el gasto en investigación y desarrollo $(I+D)$ y el nivel de patentes otorgadas a residentes, se observa en la Figura 6 que el grado de asociación entre estas dos variables es positiva, en ese sentido se puede inferir que la relación presente signos esperados en el panel estimado. Al respecto se puede anotar que a pesar de que los gastos en I+D como proporción del PIB de los paises de América latina y el Caribe no ha sido superior con respecto a las economías avanzadas ${ }^{2}$, los esfuerzos de mejora han sido significativos sobre todo cuando dichas inversiones estimulan el sector productivo para la creación de nuevos procesos o mejoras en los ya existentes.

Figura 6. Gasto en Investigación y Desarrollo como \% del PIB y su relación con el nivel de innovación

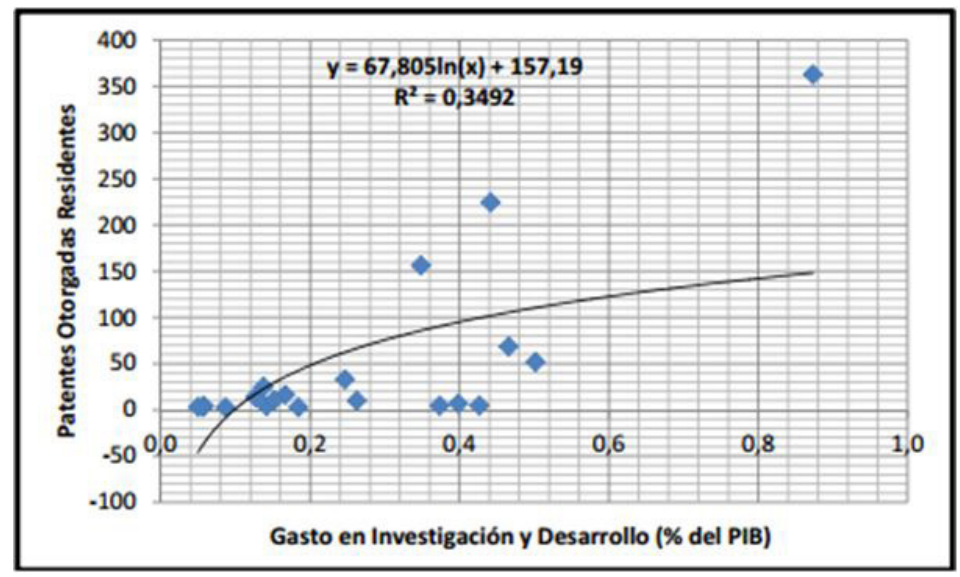

Por su parte, la facilidad de establecer negocios (Figura 7) presenta una fuerte y positiva relación con el nivel de patentes otorgadas, en este caso, varios resultados destacan la importancia del ambiente institucional para el establecimiento de negocios tendientes al incremento de patentes.

2 Según cálculos de la Red de Indicadores de Ciencia y Tecnología (RICYT), en 2009 las inversiones en ICtD de la región equivalian al 0,69\% del PIB, mientras que en 1999 representaban el 0,55\%. Durante ese mismo periodo, los paises de la OCDE aumentaron su intensidad de ICtD del 2,16\% al 2,40\%. 
Figura 7. Índice de facilidad para hacer negocios

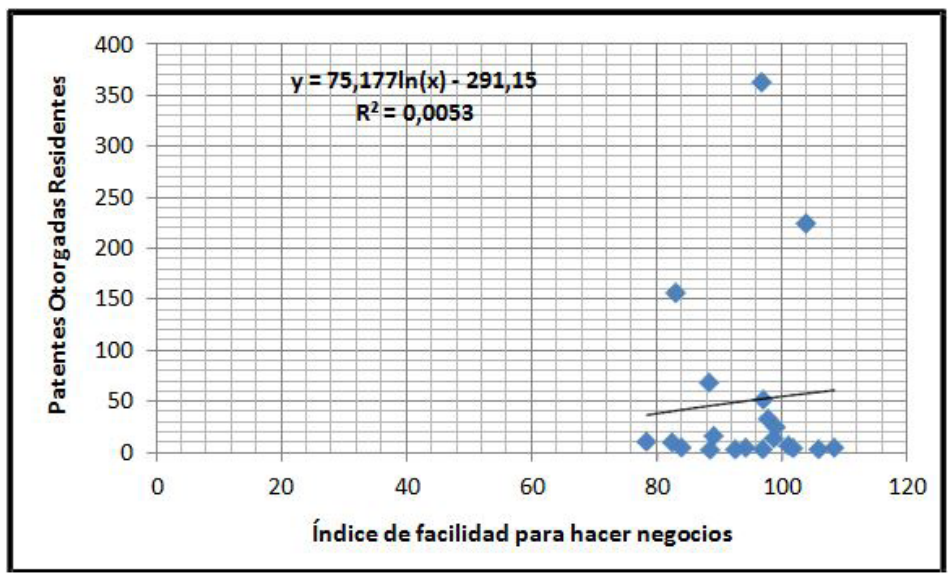

En lo referente a la relación entre la facilidad para hacer negocios que involucra dimensiones tales como el entorno regulatorio en aspectos como la apertura de una empresa, registro de propiedades y protección de inversores entre otros, se evidencia una asociación positiva con el nivel de patentes otorgadas (Figura 7), lo anterior sugiere que las actividades de innovación son susceptibles al establecimiento de reglas claras en el ambiente institucional, en ese sentido, la creación de incentivos a la protección de las innovaciones, la resolución de disputas que permitan el favorecimiento de los derechos de propiedad son fundamentales para el incremento en los niveles de innovación.

Figura 8 (a) (b).Grado de Apertura Comercial (a) y Crédito interno al Sector Privado como \% del PIB y su relación con el nivel de Innovación
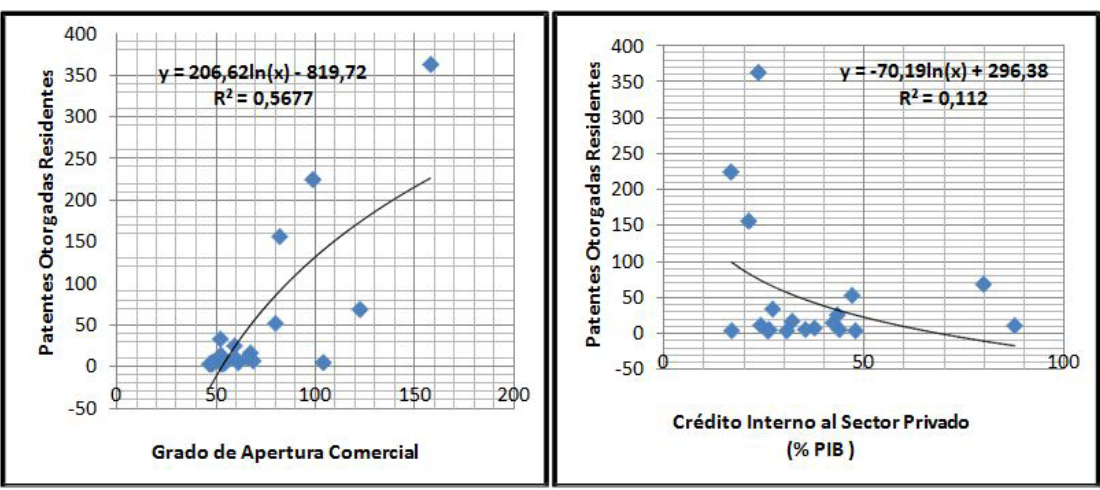
Tal y como se observa en la Figura 8 (a) el grado de apertura comercial presenta una relación positiva con las patentes otorgadas, lo anterior sugiere que entre más alto sea el grado de apertura de una economía, las empresas tenderán a proteger sus innovaciones no sólo en los mercados locales en vista de la llegada de nuevos productos sino en los mercados internacionales de los que hagan parte.

De otro lado en la Figura 8 (b) se observa que existe una relación tendencial negativa entre el crédito interno al sector privado y el nivel de patentes otorgadas, lo que denota que los niveles de crédito no han producido mejoras sustanciales en los niveles de innovación en América Latina.

\section{ESTIMACIONES ECONOMÉTRICAS}

El modelo estimado expresado en la ecuación 10 se muestra en la tabla 2. Se estimaron tres modelos, cada uno de ellos tenía como objetivo identificar de manera separada los impactos de ambiente TIC, económico y controles institucionales.

Los resultados que evidencian el impacto del ambiente TIC sobre el nivel de innovación (Tabla 2 columna 1), muestran que el coeficiente arrojado para la variable TIC (promedio de las variables banda ancha, computadores personales y celulares por cada 100 habitantes) resulta ser estadisticamente significativo en los tres modelos estimados. De tal manera que por cada abonado por 100 habitantes con suscripción en la variable TIC, el número de patentes otorgadas a residentes aumenta en $0,18 \%$. Lo que sugiere que las tecnologías de la información y las comunicaciones son imprescindibles en la actividad innovadora en la medida en que su uso y apropiación incrementa la capacidad de las empresas para innovar.

Para el modelo controlado por ambiente económico (Tabla 2, columna 2), las variables incluidas que miden el impacto del gasto en investigación y desarrollo, el crédito interno al sector privado y el grado de apertura comercial resultan ser estadisticamente significativas, esto tiene sentido cuando se encuentra que la dinámica de innovación en los países de América Latina ha estado influenciada principalmente por el gasto en $I+D$, sin embargo el crédito interno al sector privado aún sigue siendo insuficiente y en ese sentido el coeficiente que acompaña a la variable es estadísticamente significativo al 10\% únicamente, por consiguiente se puede inferir que aún hacen falta políticas que estimulen el crédito interno a las empresas de manera que este pueda explicar los niveles de innovación en los países de la región.

Tabla 2. Resultados modelo impacto de las TIC sobre el nivel de innovación

\begin{tabular}{cccc}
\hline Patentes Otorgadas & $\begin{array}{c}\text { Modelo Impacto } \\
\text { Ambiente TIC }\end{array}$ & $\begin{array}{c}\text { Modelo controlando por } \\
\text { ambiente económico }\end{array}$ & $\begin{array}{c}\text { Modelo con Controles } \\
\text { económicos e institucionales }\end{array}$ \\
VARIABLES & (1) & (2) & (3) \\
\hline
\end{tabular}




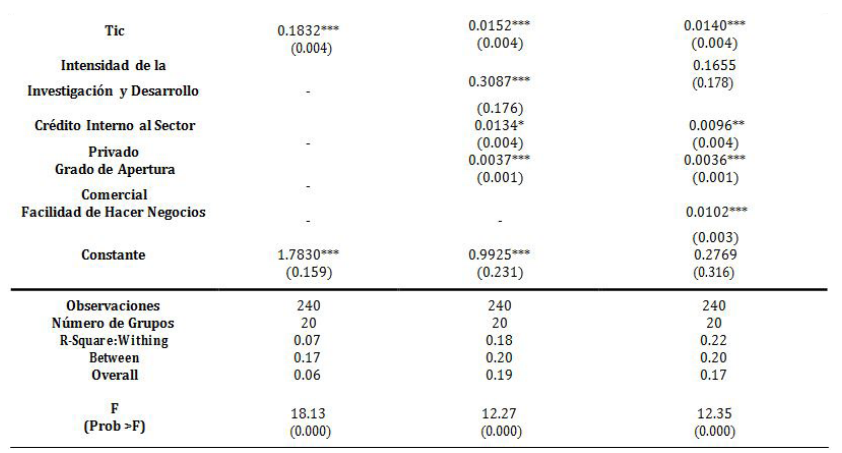

Errores estándar entre paréntesis ${ }^{* * *} p<0.01,{ }^{* *} p<0.05,{ }^{*} p<0.1$.

La variable dependiente en cada modelo está expresada en Logaritmo.

De otro lado, el modelo que incluye los controles económicos e institucionales (Tabla 2, columna 3), se observa que los coeficientes que acompañan al ambiente TIC impactan de manera positiva el nivel de patentes otorgadas, a su vez la facilidad de hacer negocios, el crédito interno al sector privado y la apertura comercial siguen siendo significativos, sin embargo, para este modelo la intensidad en investigación y desarrollo no resulta ser estadísticamente significativa a pesar de tener signo esperado (positivo).

Los tres modelos estimados presentan significancia estadística conjunta, y las variaciones al interior de grupos y entre grupos explican de manera moderada las variaciones en el nivel de otorgamiento de patentes.

En este estudio, el número inicial de paises de la muestra fue de 32. Dado que los datos que faltan son un problema común, los expertos han ideado técnicas para calcular los valores para reemplazar a ellos, trabajando a partir de variables existentes. En este trabajo se utilizó una imputación múltiple (MI). Este es un enfoque basado en el método de simulación para el análisis de datos incompletos. MI sustituye los valores perdidos con varios conjuntos de valores simulados y luego aplica análisis estándar para cada conjunto de datos completo, y ajusta los parámetros estimados (Rubin, 1987). El objetivo no es predecir los valores que faltan lo más cerca posible a los verdaderos sino para manejar los datos que faltan en una forma con una inferencia estadística válida.

\section{Conclusiones y recomendaciones}

El aumento de los niveles de innovación hoy día juega un papel fundamental en la agenda pública de los países no sólo por ser un jalonador de crecimiento económico sino porque las dinámicas favorables en innovación incrementan los niveles de bienestar social, de 
modo que las innovaciones buscan optimizar y mejorar procesos ya existentes en donde se benefician los consumidores finales en la medida en que acceden a productos de mayor calidad y con precios competitivos.

Los resultados arrojados por el modelo permiten inferir que variables como el crédito interno al sector privado contribuyen en el crecimiento del nivel de patentes otorgadas. Sin embargo, la dinámica del crédito en América Latina a diferencia de lo observado en los países desarrollados, resulta siendo marginal cuando se tienen grandes desafíos encaminados a cerrar la brecha en I+D. En efecto, se requieren medidas que incentiven la inversión pública y privada en actividades de innovación, que vinculen la esfera productiva con la I+D y que reorienten los procesos productivos con miras al incremento de los niveles de competitividad.

Se puede notar que a pesar de la importancia de la contribución de las TIC en el desarrollo de actividades de innovación, el uso de TIC por si sólo no incrementa los niveles de innovación, pues en las decisiones de innovar de las empresas confluyen otros factores de tipo institucional y económico que aportan y son imprescindibles para la actividad innovadora. Sin desconocer los esfuerzos que se han hecho en América Latina en lo que corresponde al ámbito institucional, aún se evidencia heterogeneidad en el alcance de las políticas de innovación y diferentes modelos de gobernanza de los paises de la región, lo que sugiere un fortalecimiento institucional que enfrente los retos de focalización del gasto en I+D, que se preocupe por la inserción en esquemas colaborativos regionales e incentivos a las actividades de innovación, y que articule las iniciativas que se dan en materia de innovación al interior de las economías.

Finalmente, como producto de este documento se recomienda un estudio que examine las patentes otorgadas a NO residentes en América Latina con el fin de identificar los países que están registrando innovaciones en la región, que tipo de innovaciones se registran y las razones de protección en los mercados latinoamericanos, de manera que se puedan formular estrategias que contrarrestren dicho efecto e incentiven la protección por residentes y por ende se incrementen los niveles de innovación.

\section{Referencias}

Acemoglu, D; Simon, J.; Robinson, J. (2001). "The Colonial Iringins of Comparative Development: An Empirical Investigation". American Economic Review, vol. 91, num. 5, pp. 1367-1401.

Acemoglu, D (2004). "Institutions as the fundamental Cause of Long-Run Growth". NBER Working paper, num. 10481.

Audretsch, D.B. y A.R. Thurik (2001), What is new about the new economy: sources of growth in the managed and entrepreneurial economies, Industrial and Corporate Change 10 (1), 267-315. 
Banco Mundial (2012). Indicadores [Data file]. Disponible en http://datos.bancomundial. org/indicador.

Bloom, N., Draca, M., Van, R. J., \& National Bureau of Economic Research. (2011).Trade induced technical change?: The impact of Chinese imports on innovation, IT and productivity. Cambridge, Mass: National Bureau of Economic Research.

Bloom, Nick, Mark Schankerman and John Van Reenen (2009) "Identifying technology spillovers and product market rivalry" NBER Working Paper No. 13060.

Bresnahan, T., Brynjolfsson, E. y Hitt, L. (2002). "Information Technology, Workplace Organization, and the Demand for Skilled Labor: Firm Level Evidence". Quarterly Journal of Economics. Feb 2002. pp. 339-376.

Bresnahan, T. F., Trajtenberg, M., \&t National Bureau of Economic Research. (1995). General purpose technologies: "engines of growth?". Cambridge, Mass: National Bureau of Economic Research.

Bresnahan, T. and S. M. Greenstein (1997), "Technical Progress and Co-Invention in Computing and in the Use of Computers." Brookings Papers on Economics Activity: Microeconomics, Pp. 1-78.

Broersma, L., \& Van, A. B. (January 01, 2007). ICT, "Business services and labour productivity growth". Economics of Innovation and New Technology, 16, 6, 433-449.

Brynjolfsson, E., \& Seidmann, A. (1997). Frontier research on information systems and economics. Providence, RI.

Carree, M.A. y A.R. Thurik (2003), The impact of entrepreneurship on economic growth, in: D.B. Audretsch and Z.J. Acs (eds.), Handbook of Entrepreneurship Research, pp. 437-471, Boston/Dordrecht: Kluwer Academic Publishers.

Easterly, W. y Levine, R. (1997). "Tropics, Germs and Crops: How Endowments Influence Economic Development". Journal of monetary Economics, num. 50. pp. 3-39. . (2003): "Tropics, Germs, and Crops: How Endowments Influence.

"Economic Development", Journal of Monetary Economics, vol. 50 (1), págs. 3-39.

Freeman, Chris y F. Louçâ (2002) As Time Goes By. From the Industrial Revolutions to the Information Revolution. Oxford, Oxford University Press.

Geroski, P.A., and A. Jacquemin (1985), Industrial change, barriers to mobility and European industrial policy, Economic Policy 1. Pp. 169-204.

Gershuny, J., \& Miles, I. (1983). The new service economy: The transformation of employment in industrial societies. New York: Praeger.

Griliches, Z.: R\&D and productivity: Econometric results and measurement issues. In P. Stoneman (ed.), Handbook of the Economics of Innovation and Technological Change, Basil Blackwell, Oxford, 1992

Grossman, G. y Helpman, E. (1991). Quality ladders in the theory of growth. Review of Economic Studies. 58. 43-61.

Guasch, J. L., Maloney, W. F., Perry, G., \& Schady, N. (2005). Cerrar la brecha en educación y tecnología. Bogotá, Colombia: Banco Mundial. 
Harrod, R. (1949). Toward a Dynamic Economics. McMillan.

Jaffe, A. B., \&t National Bureau of Economic Research. (1986). Technological opportunity and spillovers of R\&D: Evidence from firm's patents, profits and market value. Cambridge, MA: National Bureau of Economic Research.

Jeskanen-Sundström, Heli. (2003). ICT Statistics at the New Millennium-Developing Official Statistics-Measuring the Diffusion of ICT and its Impact. International Statistical Institute.

Maloney, W. F., \& Perry, G. (January 01, 2005). Hacia una politica de innovacion eficiente en America Latina. Revista De La Cepal (santiago De Chile), 87, 25-44.

Mankiw, G., Romer, D., y Weil, D. (1992). "A contribution to the empirics of economic growth". The Quarterly Journal of Economics. 107 (2). pp. 407-437.

Morris, M.H. (2001), Entrepreneurship is economic development is entrepreneurship, Journal of Developmental Entrepreneurship 6 (3), v-vi.

OECD (1998), Fostering Entrepreneurship; the OECD Jobs Strategy, Paris.

OCDE (2009), Patent Statistics Manual; Organización para la Cooperación y el Desarrollo Económicos (OCDE), París.

Pavitt, K., y L. Soete (1981), "International differences in economic growth and the international location of innovation, en Giersch, H., (comp.), Emerging Technologies: Consequences for Economic Growth, Structural Change and Unemployment, jcb Mohr, Tubingen.

R. Edward Freeman \&t Robert A. Phillips. Stakeholder theory: A libertarian defense Business Ethics Quarterly 12 (3):331-350 (2002)

Sanchez, A. G., \& Zayas, J. M. (January 01, 2008). Innovacion en servicios en la UE: densidad de innovacion y preeminencia economica de los innovadores. Información Comercial Española, 841, 149.

Sapprasert, K. The Impact of ICT on the Growth of the Service Industries. Contributed paper for the 2007 conference on corporate R\&D (CONCORD). Oslo: Centre for Technology, Innovation and Culture (TIK), University of Oslo.

Solow, R. (1956). "A Contribution to the theory of Economic Growth". Quarterly Journal of Economics, vol. 70, num 7, pp. 65-94.

Schumpeter, J. A. (1947). The Creative Response in Economic History. Journal of Economic History. (7). 149-159.

Swan, T. (1956). "Economic growth and Capital Accumulation", Economic Record, vol 32, num. 2, pp. 334-361.

Romer, P. (1986). "Increasing returns and long run growth". Journal of Political Economy, (1986). 94. (5). 1002-1037.

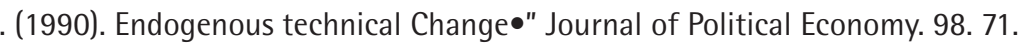

Rubin, D. B. (1987). Multiple imputation for nonresponse in surveys. New York: Wiley.

Stel, André van (2005), Entrepreneurship and Economic Growth; Some Empirical Studies, Tinbergen Institute Research Series No. 350, Erasmus University Rotterdam. 
Superintendencia de Industria y Comercio SIC (2012), "Guia de la propiedad Industrial 2020 ", Colombia.pp 28-30.

Tebaldi, E. y Elmslie, B. (2007). "Institutions, innovation and growth". Economics Seminar Series at the University of New Hampshire.

\section{Anexos}

Tabla 3. Estadística Descriptiva de las Variables

\begin{tabular}{|l|c|c|c|c|c|c|}
\hline \multicolumn{1}{|c|}{ Variable } & Notación & Media & $\begin{array}{c}\text { Desviación } \\
\text { Estándar }\end{array}$ & Mínimo & Máximo & Observaciones \\
\hline Patentes Otorgadas Residentes & Po & 50,408 & 122,158 & 1 & 952,44 & 240 \\
\hline $\begin{array}{l}\text { Suscriptores de Banda Ancha Fija } \\
\text { (por cada 100 habitantes) }\end{array}$ & fbiphp & 2,725 & 2,778 & 0 & 11 & 240 \\
\hline $\begin{array}{l}\text { Suscripciones a telefonia Móvil } \\
\text { Celular (por cada 100 habitantes) }\end{array}$ & mcsphp & 46,846 & 35,531 & 0 & 184 & 240 \\
\hline $\begin{array}{l}\text { Computadores Personales ( por cada } \\
\text { 100 habitantes) }\end{array}$ & pcphp & 56,358 & 6,916 & 12 & 61 & 240 \\
\hline $\begin{array}{l}\text { Gasto en Investigación y Desarrollo } \\
\text { (\%del PIB) }\end{array}$ & r\&dgdp & 0,275 & 0,217 & 0,03 & 1 & 240 \\
\hline $\begin{array}{l}\text { Indice de facilidad para hacer negocios } \\
\text { ( 1= más negocios amigables con la } \\
\text { regulación) }\end{array}$ & eob & 94,367 & 17,302 & 39 & 177 & 240 \\
\hline $\begin{array}{l}\text { Crédito Intemo al Sector Privado } \\
\text { (\%del PIB) }\end{array}$ & dcps & 36,775 & 20,759 & 8 & 108 & 240 \\
\hline Grado de apertura Comercial & tradeopp & 71,713 & 49,827 & 0 & 383 & 240 \\
\hline
\end{tabular}

Tabla 4. Matriz de Correlación de Coeficientes

\begin{tabular}{lrrrrrrrr}
\hline & fbiphp & $m c s p h p$ & pcphp & $r \& d g d p$ & eob & $d c p s$ & tradeopp & Po \\
\hline \hline fbiphp & 1 & & & & & & & \\
mcsphp & 0,344 & 1 & & & & & & \\
pcphp & $-0,018$ & $-0,091$ & 1 & & & & & \\
r\&dgdp & 0,137 & 0,185 & $-0,104$ & 1 & & & & \\
eob & 0,021 & 0,019 & 0,140 & 0,117 & 1 & & \\
dcps & 0,161 & 0,169 & 0,057 & 0,100 & 0,011 & 1 & \\
tradeopp & 0,482 & 0,198 & $-0,057$ & 0,488 & 0,049 & 0,122 & 1 & \\
Po & 0.194 & 0.250 & -0.141 & 0.588 & 0.232 & -0.056 & 0.584 & \\
\hline
\end{tabular}

$\mathrm{Po}=$ Patentes otorgadas residentes; fbiphp= Suscriptores de Banda Ancha Fija (por cada 100 habitantes); $\mathbf{m c s p h p = S u s c r i p c i o n e s ~ a ~ t e l e f o n i a ~ M o ́ v i l ~ C e l u l a r ~ ( p o r ~ c a d a ~} 100$ habitantes); $p c p h p=$ Computadores Personales (por cada 100 habitantes); rctdgdp= Gasto en Investigación y Desarrollo (\% del PIB); tradeopp $=$ Grado de apertura comercial; $\mathbf{e o b}=$ Índice de facilidad para hacer negocios $(1=$ más negocios amigables con la regulación); dcps= Crédito interno al sector privado (\% de PIB). 